\title{
A holographic dual to Fisher information and its relation with bulk entanglement
}

\author{
Souvik Banerjee \\ Department of Physics and Astronomy, Uppsala University, \\ SE-751 08 Uppsala, Sweden \\ E-mail: souvik.banerjee@physics.uu.se \\ Johanna Erdmenger \\ Institut für Theoretische Physik und Astrophysik, Julius-Maximilians-Universität Würzburg, \\ Am Hubland, 97074 Würzburg, Germany. \\ and \\ Max-Planck-Institut für Physik (Werner-Heisenberg-Institut), \\ Föhringer Ring 6, 80805 München, Germany. \\ E-mail: jke@mpp.mpg.de
}

\section{Debajyoti Sarkar*t}

Arnold Sommerfeld Center, Ludwig-Maximilians-University,

Theresienstr. 37, 80333 München, Germany.

and

Max-Planck-Institut für Physik (Werner-Heisenberg-Institut),

Föhringer Ring 6, 80805 München, Germany.

E-mail: debajyoti.sarkarephysik.uni-muenchen. de

In the context of relating AdS/CFT to quantum information theory, we propose a holographic dual of Fisher information metric for mixed states in the boundary field theory. This amounts to a holographic measure for the distance between two mixed quantum states. For a spherical subregion in the boundary we show that this is related to a particularly regularized volume enclosed by the Ryu-Takayanagi surface. We further argue that the quantum correction to the proposed Fisher information metric is related to the quantum correction to the boundary entanglement entropy. We discuss consequences of this connection.

Corfu Summer Institute 2016 "School and Workshops on Elementary Particle Physics and Gravity"

31 August - 23 September, 2016

Corfu, Greece

\footnotetext{
${ }^{*}$ Speaker.

${ }^{\dagger}$ This is based on a talk delivered by one of the authors at Corfu 2016 workshop. Based on ArXiv:1701.02319 [hep-th].
} 


\section{Introduction}

The theory of quantum information and measurement [1] has been a key ingredient in the understanding of the underlying quantum structure of the universe. Therefore for the last two decades significant efforts have been put to realise the connection of quantum information theory to other branches of theoretical physics. In string theory the first realisation of such connection was through the quest of understanding black hole entropy through the counting of black hole microstates [2].

Such connections were put in an even stronger footing with the advent of AdS/CFT correspondence [3]. One of the most important and crucial steps forward in this direction was the holographic realisation of entanglement entropy $[4,5]$. The proposal was to holographically compute entanglement entropy for an entangling region in a conformal field theory living on the boundary of an asymptotically AdS spacetime. The area of a minimal area codimension-two hypersurface, namely Ryu-Takayanagi (RT) surface, homologous to the said boundary region gives the corresponding boundary entanglement entropy. Interestingly, this holographic computation of boundary entanglement entropy matches exactly with the direct computation in two dimensional conformal field theory by replica trick [6]. Later on, a direct holographic justification of the RT formula was provided in [7]. On a different note, such a holographic realisation of quantum entanglement was shown to be extremely useful in understanding the structure of the dual bulk spacetime. For eternal black holes [8], quantum entanglement was found to have direct bulk interpretation in terms of regularity of the horizon [9] which turned out to be an extremely useful input in understanding black hole information paradox.

Another quantum information theory concept that has been recently studied in the context of holography is quantum complexity. In general, for a qubit systems, one way to define complexity is the number of quantum gates involved to go from a simple reference state to a complex one in a quantum circuit [1]. It is sometimes difficult to define quantum complexity precisely in mathematical terms. Within holography, this quantum complexity was first discussed in [10] where it was proposed that for thermo-field double geometries, this quantity is given by the volume of maximal volume Einstein Rosen bridge. This proposal was extended for more general mixed states in [11]. However, there is one more alternative holographic proposal for complexity given by action defined on Wheeler-de Witt patch for certain geometries [12]. Both the proposals share some common physical features such as monotonically increasing nature of complexity, though each of them has some shortcomings. While the volume proposal suffers from the fact that it introduces one new length scale, the action proposal is extremely hard to implement technically in a generic bulk space-time with null boundary surface. Recently these two concurrent proposals were reviewed and compared in more details in [13] where it was first shown that it is indeed possible to write down the action for the null boundary surface and furthermore the two definitions of complexity match up to a multiplicative constant factor dependent on the dimension of space-time.

A further concept from quantum information theory recently studied in the context of holography is the distance between two quantum states. There exist two well-accepted ways to define distance between two generic quantum states, namely (i) the Fisher information metric and (ii) the Bures metric or fidelity susceptibility $[1,14]$.

Our proposal in this work consists of two parts - first, we will propose a holographic dual to 
Fisher information metric corresponding to a spherical subregion, $R$ in the holographic CFT with $R$ corresponding to a decomposition of the full Hilbert space $H_{\text {full }}=H_{R} \otimes H_{R^{(c)}}$. In this context, we discuss a close variant of holographic complexity first introduced by [11]. We will show that a natural holographic dual of Fisher information for this mixed state is a particular regularized volume enclosed by the RT surface in the perturbed bulk spacetime.

The second part of our proposal concerns quantum corrections to Fisher information metric as defined above. Our starting point are the results of $[15,16]$, according to which the quantum corrections at order $1 / N$ to boundary entanglement entropy are equivalent to the bulk entanglement entropy. In the present paper, we propose that quantum corrections to Fisher information metric coincide with quantum corrections to the boundary entanglement entropy, the latter having an extensive volume-like behavior for particular examples as discussed in [15]. For this purpose, we argue that the reduced Fisher information is related to canonical energy (as defined in [17]), and the connection between canonical energy to the bulk modular Hamiltonian [16] is what enables us to establish this part of our proposal.

Our paper is organised as follows. In section 2, as a basis for further discussion we collect the definitions involved both on the quantum information and on the gravity dual side. In section 3 , we state in detail the two proposals put forward in this paper. We devote sections 4 and section 5 to establish these two proposals respectively. Finally we conclude in section 6 and discuss an interesting consequence and a physical consistency check of our proposal, namely the parallel between the monotonically increasing nature of holographic complexity and the classical canonical energy. We also discuss some possible questions for future work.

\section{Review of definitions}

We begin by reviewing the definitions involved in our proposals. Let us start with a pure state $\left|\psi \lambda_{i}\right\rangle$ of a quantum mechanical system characterised by the parameters, $\lambda_{i}$. Let us now perturb the parameters, $\lambda_{i}$ by $\lambda_{i} \rightarrow \lambda_{i}+\delta \lambda_{i}$. The resulting state is denoted by $\left|\chi_{\lambda_{i}}^{(\text {pert })}\right\rangle \equiv\left|\psi \lambda_{i}+\delta \lambda_{i}\right\rangle$. Now, given the two states, one can define associated density matrices defined respectively as $\sigma_{i j}=\left|\psi \lambda_{i}\right\rangle\left\langle\psi \lambda_{j}\right|$ and $\rho_{i j}=\left|\chi_{\lambda_{i}}^{(\text {pert })}\right\rangle\left\langle\chi_{\lambda_{j}}^{(\text {pert })}\right|$.

Given these two states are related by infinitesimal change in the parameter space, one can define a "distance" between the two states which takes the form [18] ${ }^{1}$

$$
F=\left|\left\langle\psi_{\lambda}(x) \mid \psi_{\lambda+\delta \lambda}(x)\right\rangle\right|=1-G_{\lambda_{i} \lambda_{j}}(\delta \lambda)^{i}(\delta \lambda)^{j}+\ldots
$$

The left hand side of (2.1) is also called quantum fidelity, which we will denote by $F$ in what follows. Also note that the $x$ dependence is absent in (2.1), when taking the inner product.

On the other hand, an equally important measure of the above-mentioned 'distance' is the quantity $G_{F, \lambda_{i} \lambda_{j}}$ which is also known as the Fisher information metric or information metric $[1,19]$. It can be defined as

$$
G_{F, \lambda_{i} \lambda_{j}}(\lambda)=\int_{x} p(x, \lambda) \frac{\partial}{\partial \lambda_{i}} \log p(x, \lambda) \frac{\partial}{\partial \lambda_{j}} \log p(x, \lambda) d x .
$$

\footnotetext{
${ }^{1}$ Note that it can very well happen that two states, which although are very close to each other are completely orthonormal. We are of course not considering such pairs. Also, the states that we consider in field theory, has a bulk gravity dual.
} 
Here $\lambda=\left\{\lambda_{1}, \cdots \lambda_{n}\right\}$ can be directly identified with the perturbative parameters discussed before. The $\lambda_{i}$ 's characterize the quantum state of the system. $n$ denotes the dimension of the statistical manifold and the $p(x, \lambda)$ are the probability functions which consist a map from a measure space $x$ to the real line. For a given pure state as above, we can identify these $p(x, \lambda)$ 's as the pure state density matrices.

For physical quantum many body systems the Fisher information metric can be understood in the following way. Let us consider a generic density matrix, $\sigma$ and perturb it by some parameter $\lambda$ which parametrizes the quantum state. Here for simplicity we assume a one-parameter family of states which however can be generalized for arbitrary number of parameters. If $\rho$ is the new density matrix corresponding to the fluctuation $\lambda \rightarrow \lambda+\delta \lambda$, then for nearby states, the density matrix $\rho$ can be expanded as

$$
\rho=\sigma+(\delta \lambda) \rho_{1}+\frac{1}{2}(\delta \lambda)^{2} \rho_{2}+\cdots,
$$

where for simplicity we have chosen the parametrization in a way such that the initial state $\sigma$ coincides with $\lambda=0 . \rho_{1}$ and $\rho_{2}$ are respective first and second order in $\delta \rho$, which is a small deviation from the reference state $\sigma$. In this case, the Fisher information metric may be defined by

$$
G_{F, \lambda \lambda}=\langle\delta \rho \delta \rho\rangle_{\lambda \lambda}^{(\sigma)}=\frac{1}{2} \operatorname{tr}\left(\left.\delta \rho \frac{d}{d \lambda}(\sigma+\delta \lambda \delta \rho)\right|_{\delta \lambda=0}\right)
$$

The notion of quantum fidelity can be extended to include mixed states as well. In terms of two density matrices $\sigma$ and $\rho$, one can rewrite the fidelity $F$ as [14]

$$
F=\operatorname{Tr} \sqrt{\sqrt{\sigma_{\lambda}} \rho_{\lambda+\delta \lambda} \sqrt{\sigma_{\lambda}}}
$$

Here $\sigma$ and $\rho$ are the density matrices associated with the parameters $\lambda$ and $\lambda+\delta \lambda$ respectively. This formula for fidelity, expressed in terms of density matrices holds true even if $\sigma$ and $\rho$ are not pure state density matrices. For pure states, the above expression becomes identical to the inner product of states as in the left hand side of (2.1). Motivated by this, we may go one step further to define a similar quantity for reduced density matrices.

As mentioned before, for a bipartite system living in a pure state $\left|\psi_{\lambda_{1}}\right\rangle$, one can define the reduced density matrix for a given subregion $R$ by partially tracing over its complement region. That is, for a pure state

$$
\left|\psi_{\lambda_{1}}\right\rangle \in H_{\text {full }}=H_{R} \otimes H_{R^{(c)}},
$$

we can compute a reduced density matrix

$$
\sigma_{R, \lambda_{1}}=\sum_{j}\left\langle\left. j\right|_{R^{c}}\left(\left|\psi_{\lambda_{1}}\right\rangle\left\langle\psi_{\lambda_{1}}\right|\right) \mid j\right\rangle_{R^{c}}
$$

where $|j\rangle_{R^{c}}$ denotes a complete set of states in the Hilbert space $H_{R^{(c)}}$. On the other hand, we can act a unitary operator on the initial state $\left|\psi_{\lambda_{1}}\right\rangle$ to get to a new pure state, $\left|\psi_{\lambda_{2}}\right\rangle$. We may then compute the corresponding reduced density matrix $\rho_{R, \lambda_{2}}$ in the new state, for the same subregion $R$. With these two reduced density matrices, we define the reduced fidelity $[14,20,11]$

$$
F_{R}=\operatorname{Tr} \sqrt{\sqrt{\sigma_{R, \lambda_{1}}} \rho_{R, \lambda_{2}} \sqrt{\sigma_{R, \lambda_{1}}}}
$$


If, however, the two states are related by an infinitesimal change in the parameter, namely $\lambda_{2}=\lambda_{1}+\delta \lambda$, (2.6) yields a perturbative expansion in $\delta \lambda$ as in the right hand side of (2.1). In this case we may identify the Bures metric as

$$
G_{R, \lambda_{i} \lambda_{j}}=\partial_{\lambda_{i}} \partial_{\lambda_{j}} F_{R}
$$

This is also known as reduced fidelity susceptibility.

In the light of $\mathrm{AdS}_{d+1} / \mathrm{CFT}_{d}$ correspondence, the gravity dual for fidelity susceptibility (as in (2.1)) was proposed in [21]. The authors considered the CFT vacuum state $\left|\psi_{\lambda}\right\rangle$ dual to pure AdS and deformed it by an exactly marginal perturbation parametrized by a single parameter, $\lambda$ to get to the state $\left|\psi_{\lambda+\delta \lambda}\right\rangle$. In the dual gravity picture this then corresponds to a Janus solution, where the pure AdS is deformed by a dilaton, with $\delta \lambda$ having the interpretation of such deformation [22].

In particular, in [21] it was shown that, for a holographic $C F T_{d}$ on $\mathbb{R}^{d}$, with marginal deformation of dimension $\Delta=d+1$,

$$
G_{\lambda \lambda}=\frac{n_{d}}{G} \frac{\operatorname{Vol}\left(V_{d-1}\right)}{\varepsilon^{d-1}}
$$

Here $G_{\lambda \lambda}$ is the fidelity susceptibility or information metric ${ }^{2}, V_{d-1}$ is the 'spatial' $(d-1)$ dimensional volume at the boundary, $G$ is Newton's constant and $n_{d}$ an $O(1)$ dimensionful constant so that the fidelity susceptibility is dimensionless. From the field theory point of view, $n_{d} / G$ is simply proportional to the central charge $C_{T}$ of the CFT in question. ${ }^{3} \varepsilon$ denotes the UV cut-off for the CFT.

So long as the reduced density matrices in the vacuum and in the excited state are commuting i.e. simultaneously diagonalizable, (2.7) is the same as the Fisher information metric corresponding to those reduced density matrices. This is automatically true for when we deal with classical states. Hence our proposal also serves as holographic dual to reduced fidelity susceptibility for these restricted class of states.

In the notation of [21], the factor of Newton's constant is hidden in the factor $n_{d}$, but we have written it explicitly here. In the boundary field theory computation, it comes from the leading order term of the boundary two-point function (in $1 / N$ expansion of a large $N$ CFT). The matching of bulk and boundary computations of fidelity susceptibility in [21] thus only involves leading order expansion in Newton's constant in both sides. In other words, the Fisher metric computed here in terms of dual gravity quantities is only the leading order semiclassical term of the full boundary Fisher information.

One can therefore naively expect an order by order expansion of $G_{\lambda \lambda}$ in Newton's constant coming from the sub-leading terms of the expansion of the two point function, as described above. We will develop this intuition in subsequent sections of this work and will eventually make use of such an expansion to relate them to corresponding parts of bulk entanglement entropy.

\footnotetext{
${ }^{2}$ [21] considered one parameter family of states so that the information metric is one- dimensional. For simplicity, we will also consider one parameter family of states.

${ }^{3}$ In fact, this order one constant $n_{d}$ is also important as this fixes the normalization of the two point function, while calculating $G_{\lambda \lambda}$ in field theory [21]. This is important for the equality as on the left hand side, generally $G_{\lambda \lambda}$ will depend on the reparametrization of $\lambda$ coordinates, whereas the volume is invariant. But essentially choosing a particular normalization of the two point function, we are fixing the reparametrization of the metric.
} 
In [21] it was further shown that the correspondence also works for the thermofield double state (TFD) [23, 8], which describes an eternal black hole in AdS in terms of two independent CFT's living on its boundary. In what follows, we will mostly focus on pure AdS state.

More recently [11] proposed a quantity called "holographic complexity" $C_{h}$ which is simply defined as

$$
C_{h}=\frac{\operatorname{Vol}(\gamma)}{8 \pi R_{A d S} G}=\frac{R_{A d S}^{d-1}}{8(d-1) \pi G} \frac{\operatorname{Vol}(R)}{\varepsilon^{d-1}} \propto C_{T} \frac{\operatorname{Vol}(R)}{\varepsilon^{d-1}},
$$

where $R_{A d S}$ is the AdS radius, $C_{T}$ is the central charge as discussed before, $\gamma$ is the codimension-2 Ryu-Takayanagi (RT) surface [4] and $G$, the Newton's constant. So, $\operatorname{Vol}(\gamma)$ denotes the volume under the RT surface. ${ }^{4} \varepsilon$ again denotes a necessary UV cut-off in field theory. On the other hand, $\operatorname{Vol}(R)$ denotes the volume of the boundary spacelike subregion $R$ which goes as $R^{d-1}$.

However, as we mentioned earlier, in this paper we consider a differential notion of holographic complexity which we call holographic complexity of formation following the idea of "complexity of formation" first discussed in [13]. One of the first problems of the proposal of [11] is that it is UV divergent and there is no reason to expect a divergent contribution to the fidelity susceptibility for a mixed state which was claimed to be the field theory dual of holographic complexity. Furthermore, the form of the divergent term is quite different from that of the fidelity susceptibility (same as Fisher information for a restricted class of states) which is known from other bulk computations and also from field theory computation in certain cases. On the contrary, as we will show, our proposal to define Fisher information and reduced fidelity susceptibility through a well defined notion of subtracted volume under RT surface will overcome both the obstacles.

It is also worth noting at this point that, as in $[10,24,25,12]$, the holographic complexity in (2.9) has been defined with a factor of Newton's constant in the denominator suggesting the fact that it is only a leading order or semiclassical contribution to the full (holographic) complexity for a subregion $R$.

\section{A proposal}

Based on these definitions, we now state the proposal which is the main message of this paper. Our proposal consists of two parts.

A. We propose an equivalence between the Fisher information metric $G_{F, \lambda \lambda}$ defined in (2.2) and a variant of the holographic complexity $C_{h}$ defined in (2.9). In the previous sections, we have already mentioned our arguments behind such a connection. In the subsequent section 4 , we provide quantitative justifications for the proposed equivalence.

B. The second part of the conjecture involves a relation between the next-order $1 / N$ quantum corrections to the Fisher information metric (or for the special set of states mentioned above,

\footnotetext{
${ }^{4}$ The RT surface is essential in computing the boundary entanglement entropy of a subregion $R$ from a holographic perspective. According to [4], the bulk counterpart of the semiclassical entanglement entropy is given by

$$
S_{E E}=\frac{\operatorname{Area}(\gamma)}{4 G}
$$

where e.g. for static geometries, $\gamma$ is the minimal area surface homologous to the region $R$.
} 
with the reduced fidelity susceptibility (2.7)) and the quantum corrections to the boundary entanglement entropy (2.10). Part A of our proposal implies that the quantum corrections to the reduced fidelity susceptibility also coincide with those to this new version of holographic complexity. Usually, the boundary entanglement entropy is defined only at the leading order in $1 / N$ expansion [4]. Here, however, we shall see that the quantum corrections to this entanglement entropy maps order by order to the quantum corrected fidelity susceptibility or quantum corrected holographic complexity. The two main properties of all of these quantities that we will focus on, are their scalings with the subregion size $R$ and their contribution at a given order in the Newton's constant $G$.

This correspondence is developed step-by-step in section 5 along with some necessary reviews of the related materials in subsection 5.1. After motivating the conjecture in the beginning of the section, in the subsequent subsections, we will justify the same first by relating the quantum correction of Fisher information to the quantum part of canonical energy (as defined in subsection 4.3) and then to the quantum part of bulk modular Hamiltonian (subsection 5.2).

\section{Proposing a holographic dual for Fisher information metric}

As mentioned above, in this section we will propose a candidate bulk dual for the Fisher information metric as a regularized volume enclosed by RT surface in the bulk. For the holographic dual picture, we consider asymptotically AdS spacetime in Fefferman-Graham coordinates. This amounts to considering states in the boundary CFT whose density matrix deviates perturbatively from that of the vacuum state with the change in boundary stress energy tensor playing the role of the perturbation parameter. In this excited state one can compute the volume under the RT surface corresponding to spherical entangling region in the boundary. This volume is generally UV divergent. However, subtracting the RT volume for the same spherical subregion in the vacuum state yields a finite, regularized result. The second order variation of this regularized volume with respect to the perturbation parameter is what we propose as the Fisher information metric. In what follows we will consider $d>2 .{ }^{5}$

To elaborate, let us consider a perturbation of $A d S_{d+1}$ given in Fefferman-Graham coordinates of the form

$$
d s^{2}=\frac{L^{2}}{z^{2}}\left[f(z) d z^{2}+\frac{1}{f(z)} d t^{2}+d \rho^{2}+\rho^{2} d \Omega_{d-2}^{2}\right]
$$

where

$$
f(z)=1+m z^{d} .
$$

$L$ is the radius of the AdS space-time.

In order to find the minimal RT surface in this perturbed AdS spacetime corresponding to a ball-shaped entangling region of radius $R$ at the boundary, we proceed by parametrizing the RT

\footnotetext{
${ }^{5}$ The $d=2$ case is a bit pathological in the sense that the perturbative expansion of the regularized volume does not contain a quadratic term. The reason is, for $d=2, h_{2}(z)$ in (4.3) vanishes identically and there is no contribution towards the minimal area surface in the second order in $m R^{d}$.
} 
surface as $\rho=h(r)$. Then, on the $t=0$ slice the RT area functional takes the form

$$
A=L^{d-1} \Omega_{d-2} \int_{\varepsilon}^{R_{t}} \frac{d z}{z^{d-1}}(h(z))^{d-2} \sqrt{f(z)+\left(h^{\prime}(z)\right)^{2}},
$$

where $\Omega_{d-2}$ is the volume of the unit $(d-2)$ sphere, given by

$$
\Omega_{d-2}=2 \frac{\pi^{\frac{d-1}{2}}}{\Gamma\left(\frac{d-1}{2}\right)} .
$$

$R_{t}$ is the turning point of the bulk minimal surface.

In order to find the minimal surface, we have to minimize the area functional (4.2) to solve for $h(z)$. It is hard to solve the equations of motion analytically. We therefore aim at solving it perturbatively in orders of $m R^{d}<<1$ and look for a solution of the form (up to linear order; quadratic order to be done later)

$$
h(z)=h_{0}(z)+m h_{1}(z)
$$

As shown in [26], this gives

$$
\begin{aligned}
& h_{0}=\sqrt{R_{t}^{2}-z^{2}} \\
& h_{1}=\frac{2 R_{t}^{d+2}-z^{d}\left(R_{t}^{2}+z^{2}\right)}{2(d+1) \sqrt{R_{t}^{2}-z^{2}}} .
\end{aligned}
$$

With these ingredients, we now move on to compute the volume under the RT minimal surface in the bulk. This is given by

$$
V_{R T}=\frac{L^{d} \Omega_{d-2}}{d-1} \int_{\varepsilon}^{R_{t}} \frac{d z}{z^{d}}(h(z))^{d-1} \sqrt{f(z)} .
$$

Our aim is now to compute the variation of this volume order by order in perturbation, $m R^{d}$ for the reasons mentioned at the beginning of this section.

\subsection{At linear order in stress-energy tensor}

From fundamentals of AdS/CFT dualities, we know that such Fefferman-Graham type expansion of AdS metric conrresponds to the corresponsding expectation values of boundary stress tensor [27]. In order to find the leading variation in the RT volume we first expand (4.3) up to leading order in $m R^{d}$

$$
h(z) \approx \sqrt{R^{2}-z^{2}}\left(1-m \frac{z^{d}\left(R^{2}+z^{2}\right)}{2(d+1)\left(R^{2}-z^{2}\right)}\right),
$$

Inserting (4.6) into (4.5) and expanding individual terms in the integral again, we have

$$
V_{R T}^{(m)} \approx \frac{L^{d} \Omega_{d-2}}{d-1} \int_{\varepsilon}^{R_{t}} d z \frac{\left(R^{2}-z^{2}\right)^{\frac{d-1}{2}}}{z^{d}}\left(1-m \frac{(d-1) z^{d}\left(R^{2}+z^{2}\right)}{2(d+1)\left(R^{2}-z^{2}\right)}\right)\left(1+\frac{m z^{d}}{2}\right) .
$$

Here the superscript $m$ denotes that this corresponds to the volume under the RT surface corresponding to perturbed geometry. Next we subtract from it the same volume for the unperturbed background, i.e for pure AdS obtained by setting $m=0$ in (4.1). This yields

$$
V_{R T}^{(m)}-V_{R T}^{(0)} \approx m \frac{L^{d} \Omega_{d-2}}{(d-1)(d+1)} \int_{\varepsilon}^{R} d z\left(R^{2}-z^{2}\right)^{\frac{d-3}{2}}\left(R^{2}-d z^{2}\right)=0,
$$


where in the first line, we have only kept terms up to order $m R^{d}$ in the integrand. Furthermore, we have replaced $R_{t}$ by $R$ since the term linear in $m R^{d}$ in $R_{t}$ gives a quadratic correction to the volume. This shows that the leading correction to the volume under RT surface vanishes identically as claimed in $[11,28]$. The vanishing of this linear term in $m$ is also crucial for our porposal (A) to work which will be clearer in the next section.

\subsection{At quadratic order in stress-energy tensor}

At quadratic order, we have

$$
f(z)=1+m z^{d}+\frac{1}{4} m^{2} z^{2 d},
$$

where coefficients of individual terms in the expansion is fixed by comparing with the FG expansion of AdS black hole. Now in order to compute the quadratic correction to the RT surface in the bulk, we start with an ansatz

$$
h(z)=h_{0}(z)+m h_{1}(z)+\frac{m^{2}}{\sqrt{R_{t}^{2}-z^{2}}} h_{2}(z),
$$

with $h_{0}$ and $h_{1}$ as given in (4.4).

The equation of minimal surface for $h_{2}$ is again obtained by extremizing the area functional, (4.2) :

$$
h_{2}^{\prime \prime}(z)+\frac{(d-1) R_{t}^{2}}{z\left(z^{2}-R_{t}^{2}\right)} h_{2}^{\prime}(z)+C_{d}(z)=0,
$$

with $C_{d}(z)$ being a complicated function of $z$. It is very hard to solve (4.10) for general dimensions. However, as an illustration, we will consider $d=3$ when (4.10) can be readily solved to yield

$$
\begin{aligned}
h_{2}(z)= & \frac{1}{320}\left(\left(160 c_{1} R_{t}-11 R_{t}^{8}\right) \log \left(z-R_{t}\right)+\left(59 R_{t}^{8}-160 c_{1} R_{t}\right) \log \left(R_{t}+z\right)\right. \\
& \left.+320 c_{1} z-\frac{20 R_{t}^{9}}{R_{t}+z}-90 R_{t}^{7} z+34 R_{t}^{6} z^{2}-30 R_{t}^{5} z^{3}+22 R_{t}^{4} z^{4}-\frac{9 R_{t}^{2} z^{6}}{2}\right)+c_{2} .
\end{aligned}
$$

$c_{1}$ and $c_{2}$ are integration constants which should be suitably chosen in order to extract the physical solution. Now one can easily note from the solution that in order to ensure

$$
\frac{h_{2}(z)}{\sqrt{R_{t}^{2}-z^{2}}} \rightarrow 0 \quad \text { as } \quad z \rightarrow R_{t},
$$

one must have

$$
c_{1}=\frac{11 R_{t}^{7}}{160} \quad \text { and } \quad c_{2}=\frac{1}{640}\left(113 R_{t}^{8}-96 R_{t}^{8} \log \left(2 R_{t}\right)\right) .
$$

Consequently, the turning point also take a new contribution at this order of perturbation and is given by

$$
R_{t}=\frac{3}{640} m^{2} R^{7}(29+32 \log (2))-\frac{m R^{4}}{4}+R
$$

Now expanding (4.5) up to quadratic order, we find

$$
V_{R T}^{\left(m^{2}\right)}-V_{R T}^{(0)} \approx \frac{21 R^{6} m^{2}}{128}
$$


Similar results can be obtained in other dimensions and we can write a general form as

$$
V_{R T}^{\left(m^{2}\right)}-V_{R T}^{(0)} \approx \frac{L^{d} \Omega_{d-2}}{d-1} \mathscr{A}_{d} m^{2} R^{2 d},
$$

with $\mathscr{A}_{d}$ a dimension-dependent constant.

This is the first central result of our paper. We see that in this way, we have arrived at a UV finite notion of regularized volume under RT surface. It is second order in $m$, the fact we will exploit to define the holographic dual to Fisher information. It is worth emphasizing that the finiteness of this quantity is critical in our proposal and what makes it meaningful for mixed states. So for now, we will go ahead and propose the holographic dual to Fisher information metric as

$$
G_{F, m m}=\partial_{m}^{2} \mathscr{F},
$$

with

$$
\mathscr{F}=\frac{\pi^{\frac{3}{2}} d(d-1) \Gamma(d-1)}{G 2^{d+1}(d+1) \Gamma\left(d+\frac{3}{2}\right) L \mathscr{A}_{d}}\left(V_{R T}^{\left(m^{2}\right)}-V_{R T}^{(0)}\right)
$$

Inserting (4.16) back into (4.15) yields

$$
G_{F, m m}=\partial_{m}^{2} \mathscr{F}=\frac{\pi^{\frac{3}{2}} d \Omega_{d-2} \Gamma(d-1)}{G 2^{d+1}(d+1) \Gamma\left(d+\frac{3}{2}\right)} L^{d-1} R^{2 d} .
$$

The choice of prefactors in (4.16) will become clear in the next section. As mentioned before, $m R^{d}$ plays the role of perturbation parameter in the dual bulk picture, in accordance with the holographic dictionary.

Although so far, (4.15) applies only to ball-shaped regions in the CFT, one may also wish to consider general entangling regions, e.g. strips. In such cases the $O\left(m R^{d}\right)$ terms do not necessarily vanish [28]. However, in principle, one can still define the holographic dual to Fisher information metric as the second order variation of the regularized RT volume with respect to the mass parameter playing the role of perturbation parameter in the dual bulk theory.

\subsection{Connection to canonical energy and boundary relative entropy}

In a related development, [29] connects the quantum Fisher information corresponding to perturbations of the CFT vacuum density matrix of a ball-shaped region to the canonical energy for perturbations in the corresponding Rindler wedge of the dual $A d S$ space-time. This is obtained by using the definition of boundary relative entropy

$$
\begin{aligned}
S_{\text {rel }}^{(\text {bdy })}\left(\rho_{\lambda^{\prime}} \| \sigma_{\lambda}\right) & =\operatorname{Tr}(\rho \log \rho)-\operatorname{Tr}(\rho \log \sigma) \\
& =\langle\log \rho\rangle_{\rho}-\langle\log \sigma\rangle_{\rho},
\end{aligned}
$$

which gives

$$
S_{\text {rel }}^{(\text {bdy })}\left(\rho_{\lambda^{\prime}}|| \sigma_{\lambda}\right)=\Delta\left\langle\mathscr{H}_{R}^{(\sigma)}\right\rangle-\Delta S_{E E} .
$$

The first term on the right-hand side denotes the change in the expectation value of the modular Hamiltonian $\mathscr{H}_{R}$ corresponding to the change in the reduced density matrix. The modular Hamiltonian corresponding to a reduced density matrix $\sigma_{\lambda}$ is defined through

$$
\sigma_{R, \lambda}=\frac{e^{-\mathscr{H}_{R, \lambda}}}{\operatorname{Tr}\left(e^{-\mathscr{H}_{R, \lambda}}\right)}
$$


Here, the second term represents the change in entanglement entropies in the two mentioned states. When the two states in question are perturbatively close to one another, expanding the density matrix $\rho_{\lambda^{\prime}}$ around $\lambda=0$ in (4.19) gives

$$
G_{F, \lambda \lambda}=\langle\delta \rho \delta \rho\rangle_{\lambda \lambda}^{(\sigma)}=\frac{\partial^{2}}{\partial \lambda^{2}} S_{\text {rel }}\left(\rho_{\lambda} \| \rho_{0}\right),
$$

where the left-hand side denotes the Fisher information metric as defined in (2.2). $\rho_{0}=\sigma$ is identified with the CFT vacuum.

Furthermore, the right-hand side of (4.21) is equal to the classical canonical energy in gravity as defined in [17], i.e,

$$
\left.\frac{\partial^{2}}{\partial \lambda^{2}} S_{\text {rel }}\left(\rho_{\lambda} \| \rho_{0}\right)\right|_{\lambda=0}=\mathscr{E}-2 \int_{\Sigma} \xi^{\mu} \frac{\partial^{2} E_{\mu v}^{g}}{\partial \lambda^{2}} v^{v}
$$

All quantities on the right-hand side of (4.22) belong to the gravity side of the correspondence. $\mathscr{E}$ is the classical canonical energy for the unperturbed vacuum state and can be expressed as an integral of boundary stress-energy tensor [17],

$$
\mathscr{E}=\int_{\Sigma} \xi^{\mu} T_{\mu v} v^{v}
$$

where $\Sigma$ is any Cauchy slice in the entanglement wedge corresponding to the ball-shaped entangling region in the boundary. $\xi$ is the conformal Killing vector, $v$ is the volume form defined as (for a $D$-dimensional spacetime)

$$
v_{v}=\frac{1}{D !} \sqrt{\tilde{g}} \varepsilon_{v, \mu_{1} \mu_{2} \ldots \mu_{D}} d x^{\mu_{1}} \wedge d x^{\mu_{2}} \ldots \wedge d x^{\mu_{D}},
$$

and $\varepsilon$ being the usual Levi-Civita tensor. $E^{g}$ denotes gravitational equations of motion with proper cosmological constant, e.g, for pure gravity in AdS

$$
E_{\mu v}^{g}=\frac{1}{16 \pi G} \sqrt{-g}\left(R_{\mu v}-\frac{1}{2} R g_{\mu v}+\frac{1}{2} \Lambda g_{\mu \nu}\right) .
$$

For the perturbed $A d S_{d+1}$ space-time as in (4.1), and for the case when the entangling region is a sphere, the canonical energy as on the right-hand side of (4.22) may be computed explicitly. One can also independently compute the second order variation of relative entropy, $\frac{\partial^{2}}{\partial \lambda^{2}} S_{\text {rel }}\left(\rho_{\lambda} \| \rho_{0}\right)$. Both calculations were done for $d=2$ in [29] and were shown to match explicitly. Again, in the holographic setup $\lambda$ is identified with the boundary energy, $m R^{d}$ where $m$ appears as a mass parameter in (4.1).

We now generalize this result to general dimensions. It reads

$$
\frac{\partial^{2}}{\partial \lambda^{2}} S_{\text {rel }}\left(\rho_{\lambda} \| \rho_{0}\right)=\frac{\pi^{\frac{3}{2}} d \Omega_{d-2} \Gamma(d-1)}{G 2^{d+1}(d+1) \Gamma\left(d+\frac{3}{2}\right)} L^{d-1} R^{2 d}=G_{F, \lambda \lambda},
$$

where in the last line we have used the definition (4.21). The basic ingredients in this computation is (4.19) and the fact that in this particular case of spherical entangling region in CFT, the modular Hamiltonian has a local expression in terms of the boundary stress energy tensor as

$$
\mathscr{H}_{R}=\int_{|x|<R} d^{d-1} x \frac{R^{2}-|x|^{2}}{2 R} T_{00}
$$


$T_{00}$ being the temporal component of the stress-energy tensor in the boundary CFT. Hence one can vary both the terms in the right hand side of (4.19) up to second order in $m R^{d}$ which yields (4.25).

Hence we find our proposal (4.17) fully consistent with the expression for Fisher information metric obtained in (4.25). This also justifies the choice of prefactors as in (4.16).

As discussed before, it is worth mentioning again at this point that for a restricted class of states when the reduced density matrices in the vacuum and in the excited state are simultaneously diagonalizable - or in other words, when the subregions are maximally entangled even after perturbation, one should expect, analogous to (4.21),

$$
G_{R, \lambda \lambda}=\frac{\partial^{2}}{\partial \lambda^{2}} S_{\text {rel }}\left(\rho_{\lambda} \| \rho_{0}\right)
$$

$G_{R, \lambda \lambda}$ being the reduced fidelity susceptibility defined in (2.6). For these states, our proposal (4.17) also serves as a holographic dual to reduced fidelity susceptibility while (4.16) can be interpreted as the holographic dual to reduced fidelity.

Let us then briefly summarize our results of this section. We show that there is a well defined, finite notion of regularized volume which serves as the holographic dual to Fisher information for two perturbatively close states. Both of them are in turn related to the classical canonical energy in the subregion. This set of connections will play a crucial role for the next part of our paper where we make statements regarding their quantum counterpart. Here we also noted that for the special class of states, all the above definitions coincide with the definition of reduced fidelity susceptibility, thus modifying the previously existing proposal of [11].

\section{Fisher information and bulk entanglement}

We now turn to the second part of our proposal on relating the $1 / N$ quantum correction to reduced fidelity susceptibility with bulk entanglement entropy.

\subsection{Bulk entanglement entropy and quantum canonical energy}

Our study on bulk entanglement entropy is motivated from a recent study in [15]. There the authors argue that the quantum correction to the boundary entanglement entropy for a boundary subregion $R$ is given by the bulk entanglement between two regions - the region inside the corresponding RT surface in the bulk and its complement. The relevant regions are depicted in figure 1. This bulk entanglement entropy can be computed order by order in Newton's constant $G$, using the replica trick in the bulk, $[7,15]$ as ${ }^{6}$

$$
S_{\text {bulk }}(R)=S_{\text {bulk }, c l}(R)+S_{\text {bulk }, q}(R),
$$

where the first term on the right-hand side of (5.1) scales as $1 / G$ (or equivalently is of order $N^{2}$ ) and corresponds to the minimal area surface term ${ }^{7}$, while the second term scales as $G^{0}$ and equals the first quantum correction to the boundary entanglement entropy.

\footnotetext{
${ }^{6}$ For the cases where one has a $U(1)$ symetry as for static black holes, it is easier to implement the replica trick for non-integer $n, n$ being the number of replicated geometries. For more general cases without any $U(1)$ symmetry, one needs to define the partition function for non-integer $n$ separately [15].

${ }^{7}$ Note that the motivation of [15] was to connect the bulk entanglement entropy with the quantum correction of boundary entanglement entropy. So, these authors only studied the $S_{b u l k, q}$ part, which they called $S_{\text {bulk-ent }}$.
} 


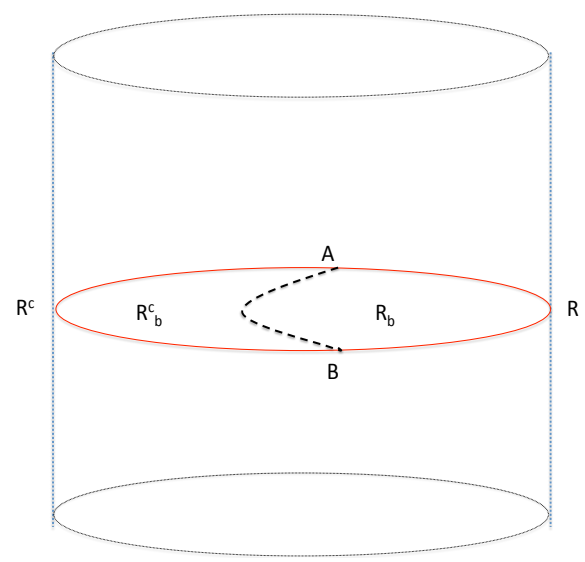

Figure 1: At the boundary $\mathrm{CFT}_{d}$ of the global $\mathrm{AdS}_{d+1}$ cylinder, we have a disc shaped region $R$ denoted by $A B$ (red line, color online). The dashed (black) line $\gamma$ represents the RT surface which divides the bulk region into two subregions $R_{b}$ and $R_{b}^{c}$. The area of this minimal surface gives the leading semiclassical term of the total boundary entanglement entropy $S_{E E}$. The $\mathscr{O}\left(G^{0}\right)$ term of bulk entanglement entropy of the region $R_{b}$ is a measure of the first order quantum correction term $S_{E E, q}$ of $S_{E E}$.

In [15], the quantum corrections to the boundary entanglement entropy $S_{E E, q}$ is given by

$$
S_{E E, q}=S_{\text {bulk }}=-\left.\partial_{n}\left(\log Z_{n, q}-n \log Z_{1, q}\right)\right|_{n \rightarrow 1},
$$

where $Z_{n}$ is the bulk partition function of the replicated geometry in the bulk.

Taking these results into account, we now proceed to state our observations. In the pathintegral language, the decomposition of (5.1) can be realized by writing the full bulk partition function $Z^{\text {bulk }}$ as

$$
Z^{\text {bulk }}=W^{\text {bulk }}+W_{\mathrm{eff}}^{\text {bulk }}
$$

where $W^{\text {bulk }}$ denotes the classical action. This gives the classical part of bulk entanglement entropy. It is essentially the same minimal area surface term that appears in Wald's treatment of the first law [30]. $W_{\mathrm{eff}}^{\text {bulk }}$ is the one-loop effective bulk action which gives $S_{\text {bulk, }}(R)$.

In the framework of replicated $n$-fold geometries $\hat{g}_{n}$, the full density matrix $\hat{\rho}_{n}^{\prime}$ is given in terms of a bulk time dependent Hamiltonian $H_{\tau}$,full which generates the time translation along the Euclideanized time $\tau$ direction [7]. That is,

$$
\hat{\rho}_{n}^{\prime}=e^{-\int_{0}^{2 \pi n} H_{b, n, \text { full }}}=e^{-\int_{0}^{2 \pi n}\left(H_{b, n, c l}+H_{b, n, q}\right)}=\hat{\rho}_{n, c l}^{\prime} \cdot \hat{\rho}_{n, q}^{\prime}
$$

where the subscripts $b, n, c l$ or $q$ above respectively suggest that the associated Hamiltonian $H$ is in bulk, in $n$-deformed spacetime and it is either classical or quantum part (at order by order in $G$ expansion). These classical and quantum parts give rise to the classical and quantum parts of the corresponding bulk entanglement $S_{\text {bulk }}$. From the above, it is most easy to see for diagonal density matrices, where by plugging in the expression (5.3) to the von Neumann formula for bulk 
entanglement entropy, it is clear that it divides the $S_{\text {bulk }}$ in classical and quantum parts as in (5.1).

$$
\begin{aligned}
S_{\text {bulk }} & =-\partial_{n}\left[\log \operatorname{Tr}\left(\hat{\rho}_{n, c l}^{\prime}\right)-n \log \operatorname{Tr}\left(\hat{\rho}_{1, c l}^{\prime}\right)\right]-\partial_{n}\left[\log \operatorname{Tr}\left(\hat{\rho}_{n, q}^{\prime}\right)-n \log \operatorname{Tr}\left(\hat{\rho}_{1, q}^{\prime}\right)\right]+\ldots \\
& =S_{\text {bulk }, c l}(R)+S_{\text {bulk }, q}(R)+\ldots,
\end{aligned}
$$

where the dots are terms which are expressed as local integrals on the RT surface. When only the background metric has a non-zero vacuum expectation value, there are two other terms that in principle can contribute to $O\left(G^{0}\right)$ correction to boundary entanglement entropy. These come from a change in area due to back-reaction on the classical background and from general higher derivative terms, respectively. ${ }^{8}$ For our present purpose we do not consider the higher derivative terms in the bulk action.

Our key observation in this section will be the term by term matching of the expansions (5.1) or (5.4) to an analogous expansion of Fisher information metric, namely,

$$
G_{F, \lambda \lambda}=G_{F, c l}+G_{F, q}
$$

Once again, the subscripts $c l$ and $q$ denote the classical and quantum parts.

To begin with, let us consider perturbation of the background metric $g^{(0)}$ by

$$
g=g^{(0)}+\delta g^{(0)}+h .
$$

Here we consider two different kinds of perturbations of the bulk metric. $h$ is an $O(\sqrt{G})$ quantum fluctuation, while $\delta g^{(0)}$ takes into account the $\lambda$ variation. Expanding the right-hand side of (4.24) in powers of $\lambda$ according to (5.6), and inserting the expansion back into (4.22), we obtain

$$
\begin{aligned}
\left.\frac{\partial^{2}}{\partial \lambda^{2}} S_{\text {rel }}^{\text {bdy }}\left(\rho_{\lambda} \| \rho_{0}\right)\right|_{\lambda=0}= & \mathscr{E}-\int_{\Sigma} \xi^{\mu} T_{\mu \nu}^{\text {grav },(2)}\left(\delta g^{(0)}\right) v^{v} \\
& -\left[\int_{\Sigma} \xi^{\mu} T_{\mu \nu}^{\text {grav },(2)}(h) v^{v}+\int_{\Sigma} \xi^{\mu} T_{\mu \nu}^{\text {matter },(2)}(g) v^{v}\right]+\text { boundary terms }
\end{aligned}
$$

This gives a clean separation of classical and quantum contributions in the Fisher metric and also the classical and quantum contributions coming towards the leading order canonical energy in the perturbed background. The first two terms on the right hand side of $(5.7)$ are classical $(\mathscr{O}(1 / G))$ contributions, with the second term arising from (4.23) upon the second order variation in $\delta g^{(0)}$. These two classical terms are the same as the right hand side of (4.22). The superscript (2) signifies the fact that all the variations are of second order in $\delta g^{(0)}$ and $h .{ }^{9}$ The remaining terms in the bracket are quantum corrections. ${ }^{10}$ Furthermore, it was shown that the boundary terms can be taken care of through a suitable choice of gauge as pointed out in [31].

Now combining (4.21) and (5.7) enables us to schematically write

$$
\left.\frac{\partial^{2}}{\partial \lambda^{2}} S_{\text {rel }}^{\text {bdy }}\left(\rho_{\lambda}|| \rho_{0}\right)\right|_{\lambda=0}=G_{F, \lambda \lambda}=G_{F, c l}+G_{F, q}
$$

\footnotetext{
${ }^{8}$ In addition to that one might need to include counter terms to have the entanglement finite.

${ }^{9}$ Note that the first order variation in either case vanishes by virtue of linearized equation of motion.

${ }^{10}$ Also note that the matter part of the stress tensor only appears at the quantum level as classically for empty AdS, the contribution is identically zero.
} 
Thus from (4.21) and (5.7), we obtain an expansion of Fisher information metric at order by order in Newton's constant and their respective connections with the classical and quantum part of the canonical energy.

Furthermore, bearing in mind (4.26), for the special case of commuting density matrices, this also implies a corresponding connection between reduced fidelity susceptibility. For the restricted class of states mentioned above, this calls for a decomposition analogous to (5.8) as

$$
\left.\frac{\partial^{2}}{\partial \lambda^{2}} S_{\text {rel }}^{\text {bdy }}\left(\rho_{\lambda}|| \rho_{0}\right)\right|_{\lambda=0}=G_{R, c l}+G_{R, q}
$$

In the next subsection, we further develop this connection, where we relate $G_{F, q}$ (and $G_{R, q}$ for the restricted class of states) to the bulk modular Hamiltonian and hence to bulk entanglement entropy.

\subsection{Canonical energy and bulk modular Hamiltonian}

Here we complete our arguments by invoking the fact that the quantum correction to canonical energy (the bracketed term in the second and third lines of (5.7)) is essentially the same as the bulk modular Hamiltonian $\mathscr{H}_{R}{ }^{\text {bulk }}$ that appears as the first quantum correction to the boundary modular Hamiltonian $[32,16]$

$$
\mathscr{H}_{R}=\frac{\operatorname{Area}(\gamma)}{4 G}+\mathscr{H}_{R}^{\text {bulk }}+\ldots
$$

This is the operator equivalent ${ }^{11}$ of the expansion of boundary entanglement entropy at order by order in $G$ expansion, namely

$$
S_{E E}=\frac{\operatorname{Area}(\gamma)}{4 G}+S_{E E, q}+\ldots
$$

Thus the results of (5.7), (5.8), (5.9), (5.10), (5.11) clearly suggest that the quantum Fisher information metric and equivalently the reduced fidelity susceptibility for the mentioned restricted class of states can indeed be understood as sum of two terms as in (5.5), namely a leading semiclassical term and a sub-leading quantum term. In this division we are simply keeping track of the orders $G^{-1}$ and $G^{0}$ respectively.

For example, if we just focus on the quantum part, i.e. the $\mathscr{O}\left(G^{0}\right)$ part, we see, from (5.8) and (5.7) that

$$
G_{F, q}=-\left[\int_{\Sigma} \xi^{\mu} T_{\mu \nu}^{\text {grav },(2)}(h) v^{v}+\int_{\Sigma} \xi^{\mu} T_{\mu \nu}^{\text {matter, }(2)}(g) \nu^{v}\right]=S_{E E, q}
$$

where the last quantity is coming from the quantum canonical energy and is equal to the modular Hamiltonian in the bulk $\mathscr{H}_{R}^{\text {bulk }}[32,16]$.

Thus following the separation in the classical and the quantum parts in (5.7), we conclude that while the classical part of the Fisher information metric $G_{F, \lambda \lambda}$ is given by the classical part of canonical energy in agreement with [29], the quantum part of it can be thought of as a dual to the bulk modular Hamiltonian. The same conclusion holds for the reduced fidelity susceptibility but only for a restricted class of states.

\footnotetext{
${ }^{11}$ This is possible by noting the connection between the entanglement entropy and the modular Hamiltonian via the density matrix as in (4.20).
} 


\section{Conclusions and outlook}

In the first part of this work we have proposed a holographic dual of Fisher information metric for a mixed state as a subtracted volume contained under the RT surface in the bulk. This also serves as a holographic dual for reduced fidelity susceptibility but for a restricted class of states, namely, when the density matrices commute before and after perturbation, i.e when the states are effectively classical. At least for this class of states we can compare our result for a previous proposal for holographic dual to reduced fidelity susceptibility in [11] in terms of holographic complexity, namely, the leading term in the volume under the RT surface. However, the proposal suffers from the following shortcomings. As we mentioned before, for classical (or effectively classical) states fidelity susceptibility is physically the same as Fisher information which is defined by the second order variation of relative entropy. Now relative entropy for a mixed state is always UV-finite and hence it is hard to justify holographic complexity which is UV-divergent being the bulk dual of this. This UV convergent behaviour was also advocated from a purely field theory computation in [33] at least for free theories and conformal field theories with large central charge. Furthermore, the second order variation of relative entropy can be explicitly computed [29, 34] and the behaviour differs significantly from that of holographic complexity as proposed in [11]. ${ }^{12}$ On the other hand, as we have shown, our proposal for the holographic dual of reduced fidelity susceptibility for those states meet both the requirements by construction. Our proposal for the bulk dual is rather similar in spirit to the recently proposed idea of complexity of formation [13, 35] which measures the relative complexity between two states. One natural question might then be whether this is also related to the computational complexity as discussed in [36, 12, 25].

One naive intuition to justify the connection to the computational complexity comes from the positivity of reduced fidelity susceptibility and the Fisher information. Furthermore, as already mentioned in [37], the identification of Fisher information with the Hollands-Wald canonical energy $[29,17]$ implies the positive energy theorem for asymptotically AdS spacetime. Our result hints at another alternative way to view the derivation of positive energy theorem for asymptotically AdS spacetimes in terms of positivity of reduced fidelity susceptibility of the mixed state. One can perhaps then interpret this positivity of canonical energy (in our case, it is the canonical energy associated to the bulk Rindler wedge corresponding to the spherical boundary region $R$ ) as a fact that we go from a reference vacuum state to a more complex excited state. In other words, the monotonically increasing nature of reduced fidelity susceptibility mimics that of the computational complexity. However, a full justification behind such a connection is yet to be understood. Work in this direction is in progress and we hope to report on this generalisation in near future. In particular, we expect a calculation within quantum field theory to reproduce the scaling with $R^{2 d}$ as in (4.17) and (4.25).

The second part of our proposal relates the quantum part of Fisher information or reduced

\footnotetext{
${ }^{12}$ However if we do not consider the complexity of formation, then one can directly compute reduced fidelity susceptibility purely within field theory by using its connection with modular Hamiltonians. In this context one can explicitly show that at the large volume limit of boundary subregions, the reduced fidelity boils down to the volume computed by [21] in the contexts of fidelity susceptibility (2.1). This can be viewed as a field theory derivation of the well-known fact that the Bures metric becomes the fidelity susceptibiility as we take the reduced density metric to be a pure state density metric.
} 
fidelity susceptibility to bulk entanglement. The latter has been argued to be instrumental in understanding the reconstruction of the bulk points inside an entanglement wedge in terms of local operators in the boundary CFT through modular evolution [16]. We expect our proposed duality might add an useful component towards a concrete study in this direction.

There are many other important issues that need to be investigated in future for a complete understanding of the proposed connections. We already mentioned about the quantum information origin of holographic complexity itself, which is missing so far in the literature. It will also be interesting to understand how our construction changes for more complicated boundary states, such as subregions with arbitrary shapes or thermofield double geometries. A generalization to multi-dimensional parameter space and a covariant generalization of our proposal also deserve a closer look.

\section{Acknowledgements}

We would like to thank Dean Carmi, Dan Kabat, Nima Lashkari for several helpful correspondence. We thank Mohsen Alishahiha and Tadashi Takayanagi for numerous helpful discussions and comments on the original manuscript. D.S also thanks the organizers of the Corfu 2016 workshop for hospitalities during the entire program. He is funded by the ERC grant "Selfcompletion".

\section{References}

[1] M. A. Nielsen and I. L. Chuang, "Quantum computation and quantum information", Cambridge university press, 2010 . .

[2] A. Strominger and C. Vafa, "Microscopic origin of the Bekenstein-Hawking entropy," Phys. Lett. B 379 (1996) 99 doi:10.1016/0370-2693(96)00345-0 [hep-th/9601029]. .

[3] J. M. Maldacena, "The Large N limit of superconformal field theories and supergravity," Int. J. Theor. Phys. 38 (1999) 1113 [Adv. Theor. Math. Phys. 2 (1998) 231] doi:10.1023/A:1026654312961 [hep-th/9711200]. .

[4] S. Ryu and T. Takayanagi, "Holographic derivation of entanglement entropy from AdS/CFT," Phys. Rev. Lett. 96, 181602 (2006) doi:10.1103/PhysRevLett.96.181602 [hep-th/0603001] .

[5] V. E. Hubeny, M. Rangamani and T. Takayanagi, "A Covariant holographic entanglement entropy proposal,” JHEP 0707 (2007) 062 doi:10.1088/1126-6708/2007/07/062 [arXiv:0705.0016 [hep-th]]. .

[6] P. Calabrese and J. Cardy, "Entanglement entropy and conformal field theory," J. Phys. A 42 (2009) 504005 doi:10.1088/1751-8113/42/50/504005 [arXiv:0905.4013 [cond-mat.stat-mech]]. .

[7] A. Lewkowycz and J. Maldacena, “Generalized gravitational entropy,” JHEP 1308, 090 (2013) doi:10.1007/JHEP08(2013)090 [arXiv:1304.4926 [hep-th]]. .

[8] J. M. Maldacena, "Eternal black holes in anti-de Sitter," JHEP 0304, 021 (2003) doi:10.1088/1126-6708/2003/04/021 [hep-th/0106112]. .

[9] M. Van Raamsdonk, “Comments on quantum gravity and entanglement," arXiv:0907.2939 [hep-th]. .

[10] D. Stanford and L. Susskind, "Complexity and Shock Wave Geometries," Phys. Rev. D 90, no. 12, 126007 (2014) [arXiv:1406.2678 [hep-th]]. .

[11] M. Alishahiha, "Holographic Complexity,” Phys. Rev. D 92, no. 12, 126009 (2015) doi:10.1103/PhysRevD.92.126009 [arXiv:1509.06614 [hep-th]]. . 
[12] A. Brown, D. A. Roberts, L. Susskind, B. Swingle and Y. Zhao, "Complexity, Action, and Black Holes,” Phys. Rev. D 93, no. 8, 086006 (2016) doi:10.1103/PhysRevD.93.086006 [arXiv:1512.04993 [hep-th]]. .

[13] S. Chapman, H. Marrochio and R. C. Myers, "Complexity of Formation in Holography," JHEP 1701, 062 (2017) doi:10.1007/JHEP01(2017)062 [arXiv:1610.08063 [hep-th]]. .

[14] A. Uhlmann, "The Transition Probability in the State Space of a* Algebra," Annalen Phys. 42, 524 (1985). .

[15] T. Faulkner, A. Lewkowycz and J. Maldacena, "Quantum corrections to holographic entanglement entropy,” JHEP 1311, 074 (2013) doi:10.1007/JHEP11(2013)074 [arXiv:1307.2892 [hep-th]]. .

[16] D. L. Jafferis, A. Lewkowycz, J. Maldacena and S. J. Suh, "Relative entropy equals bulk relative entropy," JHEP 1606, 004 (2016) doi:10.1007/JHEP06(2016)004 [arXiv:1512.06431 [hep-th]]. .

[17] S. Hollands and R. M. , "Stability of Black Holes and Black Branes,” Commun. Math. Phys. 321, 629 (2013) doi:10.1007/s00220-012-1638-1 [arXiv:1201.0463 [gr-qc]].

[18] S. L. Braunstein and C. M. Caves, "Statistical distance and the geometry of quantum states," Phys. Rev. Lett. 72, 3439 (1994). doi:10.1103/PhysRevLett.72.3439 .

[19] D. Spehner "Quantum correlations and distinguishability of quantum states", Journal of Mathematical Physics, 55, 7 (2014) http://dx.doi.org/10.1063/1.4885832 [arXiv:1407.3739 [quant-ph]]. .

[20] H. Q. Zhou, "Renormalization group flows and quantum phase transitions: fidelity versus entanglement" [arXiv:0704.2945 [cond-mat]]. .

[21] M. Miyaji, T. Numasawa, N. Shiba, T. Takayanagi and K. Watanabe, "Distance between Quantum States and Gauge-Gravity Duality,” Phys. Rev. Lett. 115, no. 26, 261602 (2015) doi:10.1103/PhysRevLett.115.261602 [arXiv:1507.07555 [hep-th]]. .

[22] D. Bak, M. Gutperle and S. Hirano, "A Dilatonic deformation of AdS(5) and its field theory dual," JHEP 0305, 072 (2003) doi:10.1088/1126-6708/2003/05/072 [hep-th/0304129]. .

[23] W. Israel, “Thermo field dynamics of black holes,” Phys. Lett. A 57, 107 (1976). doi:10.1016/0375-9601(76)90178-X .

[24] L. Susskind, "Computational Complexity and Black Hole Horizons," arXiv:1403.5695 [hep-th], arXiv:1402.5674 [hep-th]. .

[25] L. Susskind, "Entanglement is not enough," Fortsch. Phys. 64, 49 (2016) doi:10.1002/prop.201500095 [arXiv:1411.0690 [hep-th]]. .

[26] J. Bhattacharya, M. Nozaki, T. Takayanagi and T. Ugajin, "Thermodynamical Property of Entanglement Entropy for Excited States,” Phys. Rev. Lett. 110, no. 9, 091602 (2013) doi:10.1103/PhysRevLett.110.091602 [arXiv:1212.1164 [hep-th]]. .

[27] K. Skenderis, "Lecture notes on holographic renormalization,” Class. Quant. Grav. 19, 5849 (2002) doi:10.1088/0264-9381/19/22/306 [hep-th/0209067]. .

[28] O. Ben-Ami and D. Carmi, "On Volumes of Subregions in Holography and Complexity," JHEP 1611, 129 (2016) doi:10.1007/JHEP11(2016)129 [arXiv:1609.02514 [hep-th]]. .

[29] N. Lashkari and M. Van Raamsdonk, “Canonical Energy is Quantum Fisher Information,” JHEP 1604, 153 (2016) doi:10.1007/JHEP04(2016)153 [arXiv:1508.00897 [hep-th]]. .

[30] R. M. Wald, "Black hole entropy is the Noether charge," Phys. Rev. D 48 (1993) no.8, R3427 doi:10.1103/PhysRevD.48.R3427 [gr-qc/9307038]. . 
[31] S. Hollands, A. Ishibashi and D. Marolf, "Comparison between various notions of conserved charges in asymptotically AdS-spacetimes,” Class. Quant. Grav. 22, 2881 (2005) doi:10.1088/0264-9381/22/14/004 [hep-th/0503045]. .

[32] D. L. Jafferis and S. J. Suh, “The Gravity Duals of Modular Hamiltonians,” JHEP 1609, 068 (2016) doi:10.1007/JHEP09(2016)068 [arXiv:1412.8465 [hep-th]]. .

[33] N. Lashkari, “Relative Entropies in Conformal Field Theory,” Phys. Rev. Lett. 113, 051602 (2014) doi:10.1103/PhysRevLett.113.051602 [arXiv:1404.3216 [hep-th]]. .

[34] D. D. Blanco, H. Casini, L. Y. Hung and R. C. Myers, "Relative Entropy and Holography," JHEP 1308, 060 (2013) doi:10.1007/JHEP08(2013)060 [arXiv:1305.3182 [hep-th]]. .

[35] D. Carmi, R. C. Myers and P. Rath, “Comments on Holographic Complexity,” JHEP 1703, 118 (2017) doi:10.1007/JHEP03(2017)118 [arXiv:1612.00433 [hep-th]]. .

[36] A. R. Brown, D. A. Roberts, L. Susskind, B. Swingle and Y. Zhao, "Holographic Complexity Equals Bulk Action?,” Phys. Rev. Lett. 116, no. 19, 191301 (2016) doi:10.1103/PhysRevLett.116.191301 [arXiv:1509.07876 [hep-th]]. .

[37] N. Lashkari, J. Lin, H. Ooguri, B. Stoica and M. Van Raamsdonk, "Gravitational Positive Energy Theorems from Information Inequalities,” PTEP 2016, no. 12, 12C109 (2016) doi:10.1093/ptep/ptw139 [arXiv:1605.01075 [hep-th]]. . 